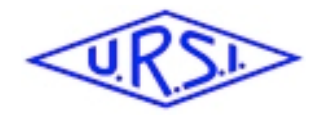

\title{
Testing of a possible RF-generator for a Space Based AOTF application in the Frame of an ESA Space Mission
}

\author{
J. Vanhamel*(1), S. Berkenbosch ${ }^{(1)}$, E. Dekemper $^{(1)}$, P. Leroux ${ }^{(2)}$, E. Neefs $^{(1)}$, E. Van Lil $^{(3)}$ \\ (1) Royal Belgian Institute for Space Aeronomy, Ringlaan 3, 1180 Brussels, Belgium \\ (2) KU Leuven, Dept. Electrical Engineering, ESAT, Advanced Integrated Sensing Lab (ADVISE), \\ Kleinhoefstraat 4, 2440 Geel, Belgium \\ (3) KU Leuven, Dept. Electrical Engineering, ESAT - TELEMIC, Telecommunications and Microwaves, \\ Kasteelpark Arenberg 10, bus 2444, 3001 Leuven, Belgium
}

\begin{abstract}
In the frame of a mission of the European Space Agency (ESA) a space qualified RF-generator needs to be developed to drive an AOTF (Acousto-Optical Tunable Filter) to make hyper-spectral images of the limb of the Earth. Custom off-the-shelf electronics can not fulfil the requirements for this scientific mission, hence alternative solutions for the RF-generation are investigated. This paper describes one of the possible solutions.
\end{abstract}

\section{Introduction}

Several architectures have been developed to produce a high quality RF-signal largely depending on the application. The use of commercial off-the-shelf electronic components is not allowed for space applications. Therefore space-qualified components have to be used. Several possible solutions were investigated in the framework of the ALTIUS mission (Atmospheric Limb Tracker for the Investigation of the Upcoming Stratosphere) [1, 2]. The goal of this mission is to measure atmospheric trace species concentration profiles with a high spatial resolution [3]. The concept relies on the acquisition of spectral images of the bright atmospheric limb at specific wavelengths. Three independent channels will be implemented inside the instrument, each working in a different spectral range, namely ultraviolet (UV): 250 to $400 \mathrm{~nm}$, visible (VIS): 440 to $800 \mathrm{~nm}$ and near-infrared (NIR): 900 to $1800 \mathrm{~nm}$. In two of the three channels, a wide aperture $\mathrm{TeO}_{2} \mathrm{AOTF}$ will be used for the selection of different spectral wavelengths. The working principle of an AOTF is based on the interaction of sound and light inside a birefringent crystal $[4,5]$. The RF-power is converted into acoustic power by means of a piezoelectric transducer. For every specific RF-frequency and RF-power, only photons of particular energy will interact with the sound wave inside the crystal. The diffracted beam exits under a different angle than the rest of the light. The beam of interest is focused onto a detector which performs the spectral imaging.

The RF-chain consists of an RF-generator, coupled to an RF-amplifier (Figure 1). The output of the RF-amplifier is applied to the transducer of the AOTF via a matched impedance network.

Since every independent channel works in a predefined spectral range, the applicable RF-frequency band per channel is predetermined. For the VIS channel: 60 to 120 $\mathrm{MHz}$, the NIR channel: 30 to $60 \mathrm{MHz}$ and the UV channel: 130 to $260 \mathrm{MHz}$. For the UV channel, the AOTF would have been made from a KDP crystal (potassium dihydrogen phosphate). Unfortunately the necessary level of maturity for a space mission was not reached for this type of AOTF. Instead the selection will be done by a Fabry-Pérot system. Nevertheless, the investigation on how to develop a high frequency RF-chain in the UV up to flight level is interesting and will be pursued.

For the design of a space-qualified RF-generator different approaches were investigated $[1,2]$. Two solutions were withheld based on previous results $[1,2]$. The first solution uses a PLL (Phase Locked Loop), while the second solution is based on DDS (Direct Digital Synthesis) inside a space qualified FPGA (Field Programmable Gate Array). Only the first solution will be further discussed in this paper.

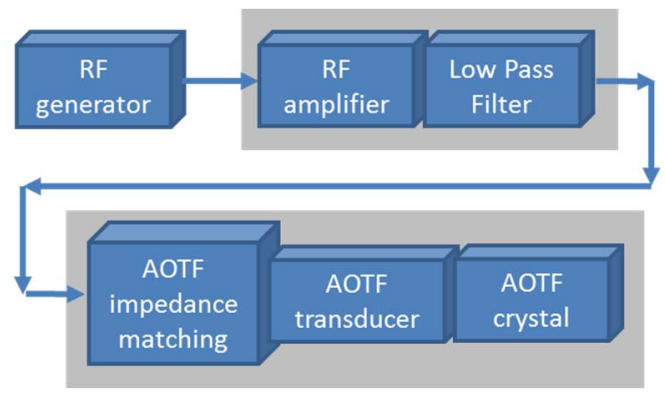

Figure 1. Channel RF-chain concept

\section{RF-generator development}

The development of the RF-generator will focus on survivability in space and compliance with the selected spacecraft and launcher. To achieve this, a well-defined list of environmental tests has to be carried out, such as thermal-vacuum, radiation, vibration, shock and EMC (Electro-Magnetic Compatibility). The test levels are derived from the expected environment the instrument 
will have to operate in. The selection of electronic components will be done in accordance with spacequalified standards. ESA restricts the use of these components to those listed in their preferred parts list. Because the project is currently in a preliminary design phase, some requirements still need to be defined. These not-defined parameters will depend on the choice of launcher and the design of the platform. This paper will focus on the fulfilling of the currently available requirements listed in Table 1.

Table 1. Preliminary design parameters

\begin{tabular}{|l|l|}
\hline \multicolumn{1}{|c|}{ Requirement } & \multicolumn{1}{c|}{ Value } \\
\hline UV-channel frequency range & $130-260 \mathrm{MHz}$ \\
\hline VIS-channel freq. range & $60-120 \mathrm{MHz}$ \\
\hline NIR-channel frequency range & $30-60 \mathrm{MHz}$ \\
\hline $\begin{array}{l}\text { Unwanted spectral } \\
\text { components in RF-output }\end{array}$ & $<-30 \mathrm{dBc}$ \\
\hline Nominal load & $50 \mathrm{Ohm}$ \\
\hline RF-generator accuracy & $1 \mathrm{kHz}$ \\
\hline RF long term freq. stability & $5 \mathrm{kHz}$ \\
\hline Phase Noise & $<-120 \mathrm{dBc} / \mathrm{Hz}$ \\
& $@ 10 \mathrm{kHz}$ \\
\hline RF resolution & $5 \mathrm{kHz}$ \\
\hline RF-generator output power & $>0 \mathrm{dBm}$ \\
\hline
\end{tabular}

The PLL-solution has already been breadboarded and tested [1, 2] but the different components are now integrated onto one custom-designed PCB (Printed Circuit Board) for the UV-channel. This integration will have an impact on the stability and performance of the solution.

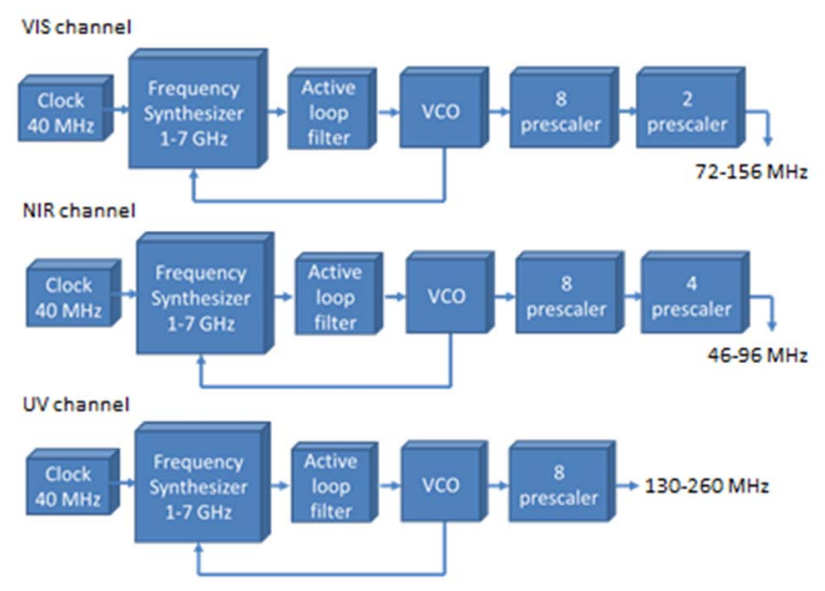

Figure 2. Channel PLL solution setup

The core of the PLL-based RF-generator solutions for the three channels (Figure 2) is based on a space-qualified frequency synthesizer of Analog Devices (ADF4108S) [6], combined with a VCO (Voltage Controlled Oscillator) of Minicircuits (ZX95-2500W+) [7], an inhouse custom-designed active loop filter and a spacequalified divider of Peregrine Semiconductor/E2V [8]. The VCO is controllable between $1 \mathrm{GHz}$ and $2.4 \mathrm{GHz}$.
The VCO flight model is not selected yet, but will have the same characteristics as this engineering model.

Only the UV-channel is currently designed as an integrated PCB (Figure 3). The shielded part of the PCB represents the RF-generator. The extra superposed PCB on the right is an 8-bit PIC microcontroller (PIC18F46J50) of Microchip [9] and is used to program the different parameters in the ADF4108 related to the different mission scenarios. For this a self-developed GUI (graphical user interface) is foreseen as well. The power handling is currently done by the PCB itself but will be externally provided in a later stage.

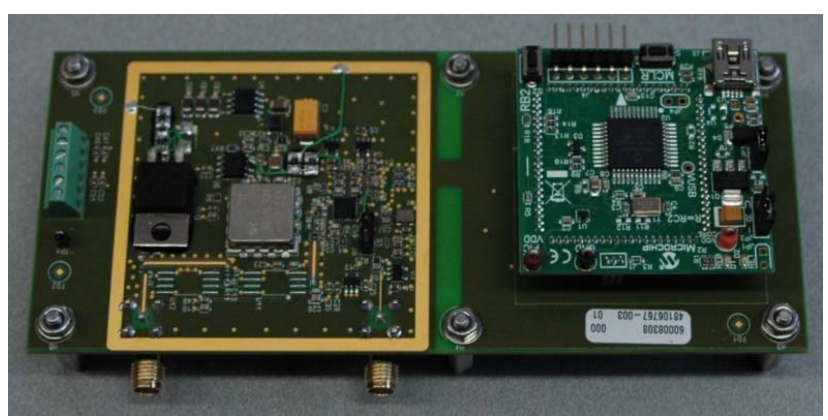

Figure 3. PCB of the UV-channel

The only difference between the UV-channel and the two other channels is the use of an additional prescaler at the end of the chain (Figure 2). Both additional prescalers are available as a space qualified product [8]. It is expected that this extra division will have an impact on the unwanted spectral components in the RF-output. This will not be discussed in this paper but will be investigated in a later stage.

In the next paragraph the compliance with the design parameters (Table 1) will be examined for the UVchannel design, i.e. without an additional $\div 2$ or $\div 4$ prescaler.

\section{Measurement results}

\subsection{Spectral output}

Different frequencies are programmed into the ADF4108. The generated output spectrum is compliant with the requirements for the complete frequency range: no excessive harmonics or spurs exist (Figure 4). The level of the second harmonic is less than $-21 \mathrm{dBc}$, the third harmonic is less than $-10 \mathrm{dBc}$. The results impose the use of an additional passband filter for suppressing the higher harmonics to a level of $-30 \mathrm{dBc}$. As a consequence, it is expected that the output power level will be slightly attenuated. 


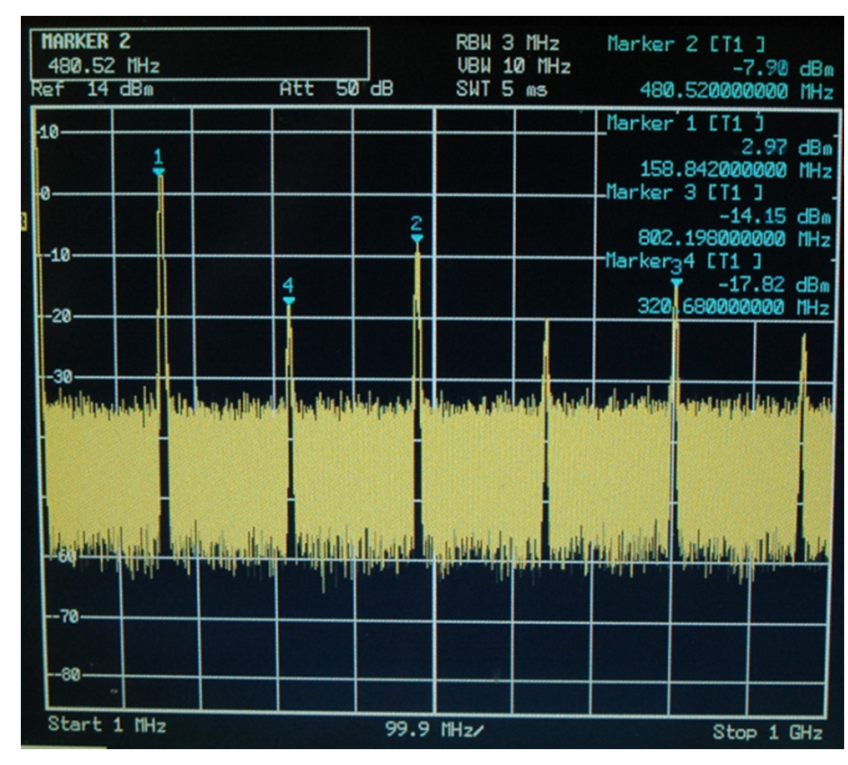

Figure 4. Spectral content generator output

\subsection{Spectral resolution}

In Table 1 it can be found that a spectral resolution of $5 \mathrm{kHz}$ is required. A frequency of $1200 \mathrm{MHz}$ is programmed into the ADF4108. After division by 8 the output frequency is $150 \mathrm{MHz}$ (Figure 5). By adjusting the programmed frequency to $1200.04 \mathrm{MHz}$ the output frequency shifts to $150.005 \mathrm{MHz}$ (Figure 6). This is compliant with the requested resolution step size of 5 kHz.

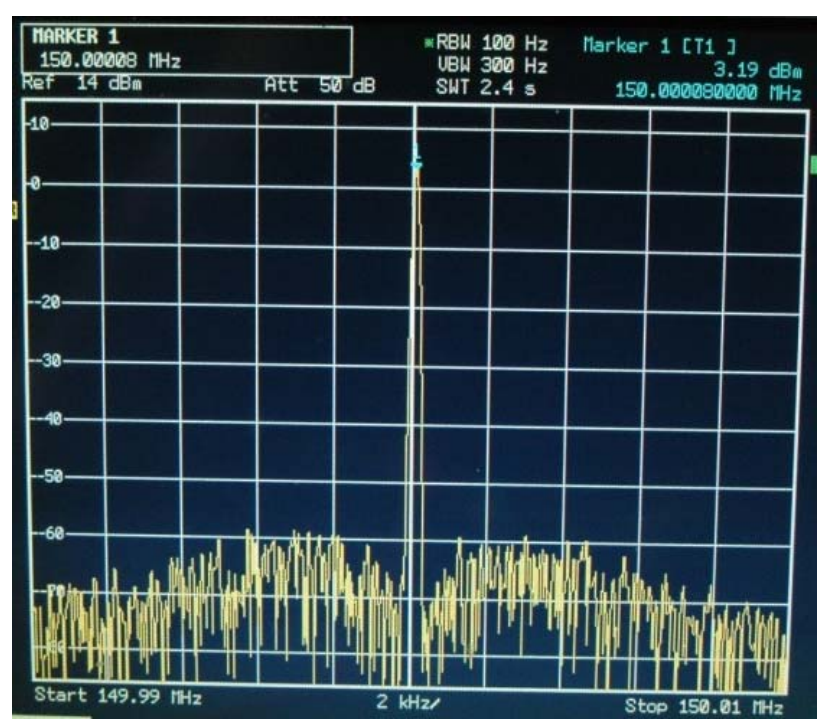

Figure 5. Spectral resolution of $5 \mathrm{kHz}$, step 1

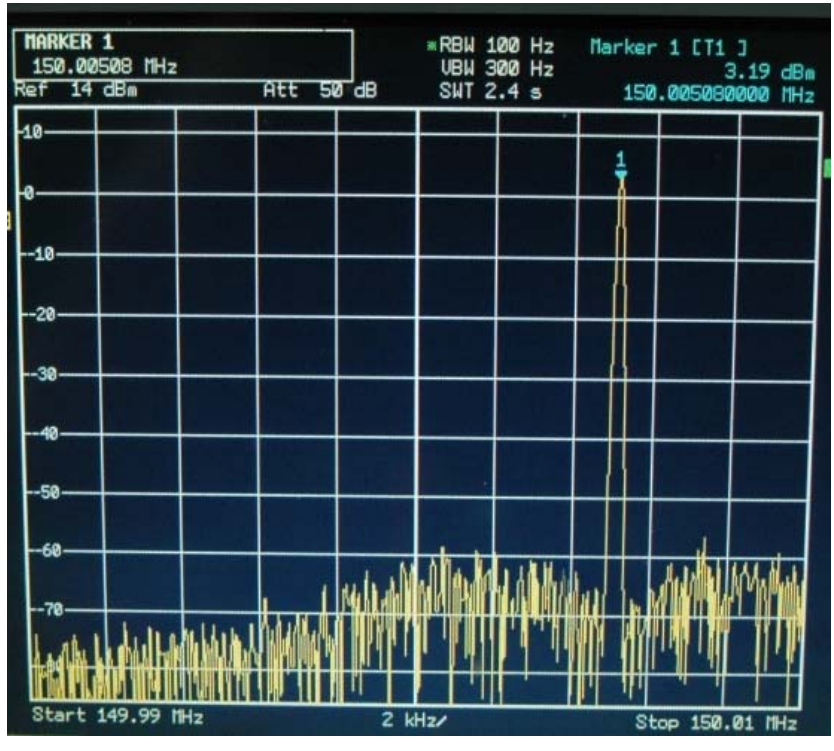

Figure 6. Spectral resolution of $5 \mathrm{kHz}$, step 2

\subsection{Output power}

Measurements have been carried out to check the output level of the RF-generator. Again different input frequencies are programmed into the ADF4108, taking into account the required output range. The output level measured after the $\div 8$ prescaler varies with the output frequency. The lowest output level measured is $+2.5 \mathrm{dBm}$, the highest is $+3.3 \mathrm{dBm}$ (Figure 7). The output level for the overall frequency range is above the requested $0 \mathrm{dBm}$.

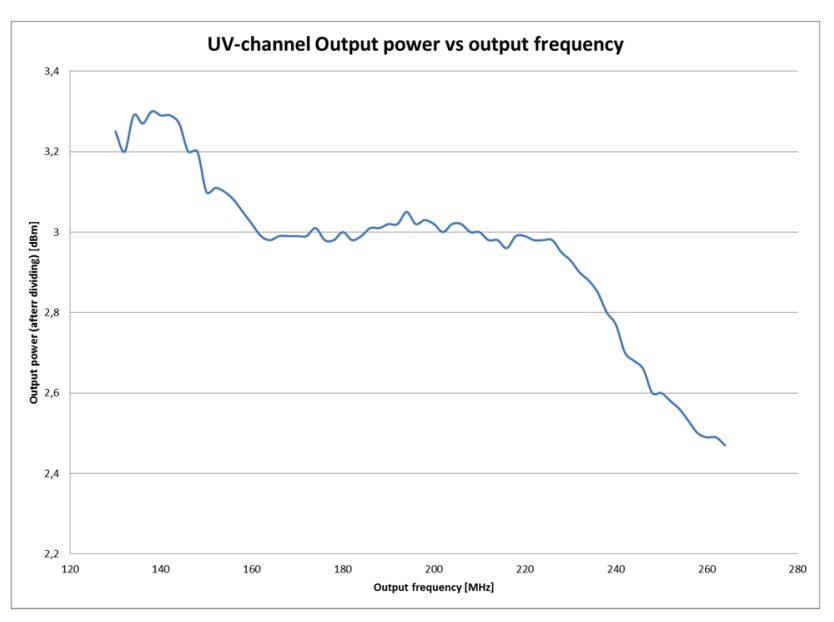

Figure 7. Output power level UV-channel

\subsection{Other parameters}

Other tests were carried out to check the other parameters listed in Table 1. The RF long term frequency stability has to stay within $5 \mathrm{kHz}$ in a time interval of 100 seconds. The lower limit, the higher limit and the center frequency of the UV frequency band were programmed into the ADF4108 to check if this requirement is met. The deviation of the RF output signal depends strongly on the drift of the reference oscillator. The selected part has a 
drift of 1 ppm per degree Celsius. The final space qualified reference oscillator will have the same characteristics as this engineering grade component. Taking this value into account, the RF-generator output drift is limited and well within the requested drift range.

To measure the phase noise, the SSB (Single Side Band) method is used. For this a R\&S 1093.4495.03 Spectrum Analyser is used. Phase noise is measured at an offset of $10 \mathrm{kHz}$ related to the carrier. Again measurements were done at the lowest, highest and center frequency of the UV frequency band. For an output frequency of $130 \mathrm{MHz}$ the phase noise at $10 \mathrm{kHz}$ offset is $-101 \mathrm{dBc} / \mathrm{Hz}$. At 200 $\mathrm{MHz}$ the phase noise is $-101 \mathrm{dBc} / \mathrm{Hz}$. Finally at $260 \mathrm{MHz}$ the phase noise is $-102 \mathrm{dBc} / \mathrm{Hz}$. These values indicate that the proposed RF-generator manages to fulfill the phase noise requirements (Table 1).

\section{Conclusion and future work}

The RF-generator presented in this paper is part of the development of a space borne remote sensing instrument (ALTIUS). The development of an RF-driving mechanism for the UV-channel is taken as a baseline because the generation of high stable frequencies in the harsh environment of space is a major challenge. Using a space qualified PLL-approach for the design of a frequency sweeping RF-generator allows then further downscaling of the frequency for the VIS- and NIRchannel.

With the help of a merged PCB design prototype, different parameters were checked. To reach the requested $-30 \mathrm{dBc}$ for the harmonics and spurs, an additional filter needs to be implemented in the RF-chain. This will have an impact on the final output power level of the RFgenerator and will be investigated in a later phase. Currently the output power of the RF-generator is around $+2.9 \mathrm{dBm}$ which is well above the requested $0 \mathrm{dBm}$. Also the spectral resolution of $5 \mathrm{kHz}$ as well as the stability of $5 \mathrm{kHz}$ in 100 seconds can be achieved with the current design. The phase noise is also within specification. Currently tests have been performed on the UV-channel only. Adaptations are needed for the VIS- and NIRchannel. Research is ongoing to check if the implementation of two additional prescalers at the end of the RF-generator has an impact on the performance in VIS and NIR. Thermally-induced jitter is also under investigation, a possible issue when using an analog VCO circuit.

\section{Acknowledgments}

The ALTIUS project is supported by the Belgian Federal Science Policy Office (BELSPO) and funded through ESA-PRODEX contract PEA4200090274.

\section{References}

[1] J. Vanhamel et al., "RF-driving of Acoustic-Optical Tunable Filters; Design, Realization and Qualification of Analog and Digital Modules for ESA", Microelectronics
Reliability, 55 (2015), pp. 2103-2107, doi: 10.1016/j.microrel.2015.07.034

[2] J. Vanhamel et al., "Implementation of Different RFchains to Drive Acousto-Optical Tunable Filters in the Frame of an ESA Space Mission", Radio Science Bulletin, 357 (June 2016), pp. 37-43

[3] E. Dekemper et al., "ALTIUS: a Spaceborne AOTFbased UV-VIS-NIR Hyperspectral Imager for Atmospheric Remote Sensing", Proceedings Of SPIE, 9241-92410L(1-10), 2014

[4] I.C. Change, "Noncollinear Acousto-Optic Filter with Large Angular Aperture", Applied Physics Letters 25, 1974, pp. 370-372, doi: http://dx.doi.org/10.1063/1.1655512

[5] E. Dekemper, "Development of an AOTF-based hyperspectral imager for atmospheric remote sensing", Ph.D. Thesis, UCL, Louvain-la-Neuve, Belgium, pp 99136,2014

[6] Information about the ADF4180S,

www.analog.com/en/products/application-

specific/militaryaerospace/aerospace/adf4108s.html

[7] Information and datasheet VCO ZX95-2500W+, http://194.75.38.69/pdfs/ZX95-2500W+.pdf

[8] Information and datasheets about the space qualified prescalers

http://www.e2v-

us.com/products/semiconductors/peregrinerf/

[9] Information and datasheet of the Microchip PIC18F46J50 8-bit microcontroller http://www.microchip.com/wwwproducts/en/PIC18F46J5 0 